
no le llegó la noticia de la existencia de un cuarto manuscrito. Su descubridora, Olga Omatos, no refiere en ningún momento ni la biblioteca donde se encuentra ni número de inventario, signatura o catálogo. En el presente trabajo, siguiendo el hilo del análisis de la edición de Kejayoglu, publicamos estos datos, así como aportamos otros nuevos en relación a la datación este nuevo manuscrito.

PALABRAS CLAVE: Quijote, griego moderno, traducción manuscrita, edición impresa.

\title{
A propósito de la edición de la primera traducción griega del Quijote y el hallazgo de un nuevo manuscrito*
}

When Georgios Kechagioglou made his philological edition of the first greek translation of Don Quixote, initially transmited in three manuscript (athinean collection Sgourdaios, n. 1 of Vitina Municipal Library and n. ${ }^{\circ} 1471$ of the Romanian Academy Library), he didn't know the existence of a fourth manuscript. The discoverer, Olga Omatos, don't comunicate neither the library where it is, neither any call number. In this work, following the thread of the analysis of the Kechagioglou edition, we publish these informations and contribute

Guillermo Marín Casal

Grupo de Investigación de Estudios

Bizantinos y Neogriegos. CSIC another new matters related to dating this new manuscript.

KEY WORDS: Don Quixote, modern greek, handwritten translation, printed edition. 
El catedrático de Filología Neogriega en la Universidad Aristóteles de Salónica Yeoryos Kejayoglu y su colega Ana Tabaki, profesora en la 164 Universidad Nacional Capodistrias de Atenas y colaboradora científica en el Instituto de Investigaciones Científicas Neogriegas de la Fundación Nacional de Investigaciones Científicas de Grecia, publicaron en diciembre de 2007 la edición crítico-filológica de la primera traducción conocida a día de hoy de El ingenioso hidalgo don Quijote de la Mancha. El voluminoso ejemplar que nos of recen estos dos neohelenistas especializados en la recepción, circulación y traducción de clásicos literarios europeos occidentales en el ámbito griego de los siglos XVIII y XIX, se abre con una harto exhaustiva introducción de I50 páginas, que preludia el texto editado por Y. Kejayoglu (Kejayoglu-Tabaki, 2007: I-632). E1 volumen consta también de un imprescindible glosario (Kejayoglu-Tabaki, 2007: 635-812) de vocablos que se documentan en el texto, pero ya desusados en griego moderno común actual. Por último, cierran el libro 28 láminas facsímiles, que reproducen algunos folios de tres de los cuatro manuscritos que transmiten esta traducción, así como algunas ilustraciones copiadas a tinta en una de estas copias manuscritas.

Gracias al trabajo de estos excelentes filólogos el público griego actual tiene la posibilidad

El presente trabajo se enmarca en el proyecto de investigación Lengua, literatura y sociedad en comunidades islamo-cristianas del Mediterráneo (FFI2008-06919-CO2-OI) financiado por el Ministerio español de Ciencia e Innovación de España y dirigido por el Dr. Pedro Bádenas de la Peña, cuyas sugerencias y correcciones agradecemos desde aquí. Asimismo queremos expresar también nuestra gratitud hacia el director del Instituto Cervantes, Dr. Eusebi Ayensa Prat, por hacernos llegar la fotografía del colofón de este nuevo manuscrito, y hacia el Dr. Yeoryos Kejayoglu, quien tuvo la amabilidad de facilitarnos un ejemplar de su reciente edición de la primera traducción griega del Quijote, así como su breve, pero sustancial reseña sobre el último de los manuscritos que la transmiten.

de leer, por primera vez en formato de libro impreso, la que es no sólo la primera traducción griega del Quijote, sino también la primera versión de la universal obra de Cervantes concebida en sureste europeo, anterior incluso a la primera traducción rusa ( 1769 ). El texto inédito hasta ahora, transmitido sólo de forma manuscrita y fragmentaria, adelanta en más de un siglo el primer Quijote impreso en lengua griega (Esmirna 1852), obra de Ceódoros Catramís, quien como bien indica el título de su traduc-

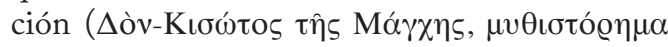

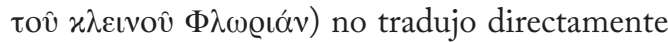
el original español de Cervantes, sino la versión francesa de Jean-Pierre Claris de Florian (París I799), tan en boga a comienzos del siglo XIX ${ }^{\mathrm{I}}$.

Las coordenadas espacio-temporales (Kejayoglu - Tabaki, 2007: $\left.{ }^{*}{ }^{2}{ }^{*}{ }^{*} 66\right)$ se sitúan en la tercera década del siglo XVIII, en Bucarest, por aquel entonces capital del principado danubiano de Valaquia, gobernado a la sazón por el voivoda fanariota ${ }^{2}$ Nicolás Mavrocordatos, en cuya corte frecuentada por intelectuales áulicos griegos y grecohablantes se gestó esta primera traducción griega manuscrita de la obra maestra

I Ésta es realmente la primera traducción impresa griega del Quijote y no la versión del esmirneo Isidoridis Skilitsis (Trieste I864), la cual parte, como la anterior, de la versión intermediaria de Florian, que constituye el texto de partida de todas las traducciones griegas publicadas hasta 1919. Sobre estas traducciones, así como la fortuna del Quijote en las letras y la cultura griegas pueden consultarse en español los trabajos de Alicia Villlar, Victor Ivanovici y la revisión y actualización que yo mismo he realizado «Tras las huellas griegas de Don Quijote en sus andanzas por Bucarest, Esmirna, Trieste, Constantinopla, Nueva York y Atenas» de próxima aparición en la revista norteamericana Cervantes.

2 Fanariota se refiere a todo aquel que procede del barrio constantinopolitano (o estambulí) del Fanar (del gr. Фavóøı(ov) 'fanal, farol'), donde se encuentra, desde 1586, la sede del Patriarcado Ecuménico, en cuyo seno surgieron una serie de familias aritocráticas que coparon y monopolizaron buena parte de la cancillería y de la diplomacia del Imperio otomano. 
cervantina. Éste es, pues, el primer testimonio de recepción y difusión del Quijote por los Balcanes y la Rumelia otomana, tierras ignotas hasta entonces para el universal héroe manchego, que a lo largo del siglo XVIII constituyen un campo fértil para la asimilación de elementos culturales (y literarios) procedentes tanto del oriente como del occidente europeos.

En este amplio marco de los principados danubianos de Moldavia y Valaquia circularon, al menos, cuatro copias manuscritas, que transmiten fragmentariamente el texto de esta primera traducción griega del Quijote. Hasta hace relativamente poco se desconocía la existencia de cualquiera de ellas. Apenas en 1966 Lukía Drulia descubrió, dentro de la biblioteca familiar de la familia ateniense Sgurdeos, un primer manuscrito de la traducción. El escaso texto que transmitía comprendía desde el último tercio del capítulo I hasta el capítulo XIV, inclusive, de la primera parte del Quijote, y su datación hemos de acotarla entre los años I72I y 1745 . Tras este primer manuscrito, han aparecido desde 1966 tres más que transmiten otros fragmentos y pasajes de la traducción. A mediados de la década de r980 Y. Kejayoglu (I990) localizó e identificó (ff. $26 \mathrm{I}^{\mathrm{r}}-3 \mathrm{I2}^{\mathrm{v}}$ ) la traducción griega de la historia del «Curioso impertinente» (Quijote I, 33-35) en el códice misceláneo n. ${ }^{\circ}$ I de la biblioteca municipal de Vitina (Arcadia, Peloponeso), cuyo propietario original, el poeta Constandinos (Cesáreo) Dapontes, hubo de copiar el texto entre los años I73 y y 765 durante su larga estancia en los principados danubianos. Una década después la profesora Ana Tabaki (I997 y 1999) halló en la Biblioteca de la Academia Rumana en Bucarest el códice griego n. ${ }^{\circ}$ I47I, acéfalo y mútilo, que en sus 530 folios transmite, casi completa, la Segunda Parte del ingenioso caballero Don Quijote de la Mancha, salvo los primeros folios del principio y los últimos del final, de los capítulos I y LIV respectivamente. La datación de este manuscrito se sitúa entre la cuarta o quinta década del siglo XVIII, de modo que éste constituye la copia más cercana de la traducción original. Asimismo el códice contiene cinco copias a tinta de los grabados que acompañaban la tercera edición impresa de la traducción italiana de Lorenzo Franciosini L'ingegnoso Cittadino Don Chisciotte della Mancia (Roma 1677), que constituye, sin lugar a dudas, la fuente de la que parte esta primera traducción griega manuscrita del Quijote.

No obstante, en los últimos años ha salido a la luz un cuarto manuscrito, cuya existencia desconocía Y. Kejayoglu, cuando éste establecía el texto de su edición, por lo desafortunadamente no pudo ser incluido en ella. Además la investigadora que estudió y estudió por primera vez el manuscrito, Olga Omatos, pese a la descripción que ofrece del manuscrito y a las fotografías que adjunta al final de su artículo (Omatos, 2006: I7I-I72, I82-I85) no identifica en ningún momento este nuevo manuscrito ni tampoco refiere ni la biblioteca en que se encuentra. ${ }^{3}$ Afortunadamente estos datos los aporta Y. Kejayoglu en una breve nota publicada muy recientemente (2009): se trata del manuscrito n. ${ }^{\circ} 798$ de la Biblioteca Yenadios de Atenas, el cual fue descubierto durante la limpieza e inspección de un desván de esta biblioteca ateniense.

Este nuevo manuscrito es acéfalo y mútilo, pero consta de 597 folios, en los que transmite más texto de la traducción que el resto de copias, desde la «Historia del cautivo» (Quijote

3 Asimismo, según se desprende de su artículo, $\mathrm{O}$. Omatos también desconocía la existencia del manuscrito n. ${ }^{\circ}$ I47I de la Biblioteca de la Academia Rumana de Bucarest, así como el proyecto de la edición de los tres primeros manuscritos que se estaba realizando desde hacía tiempo en Grecia. 
I, 39), ya iniciada, hasta el final de la segunda parte. En su colofón, del que adjuntamos una fotografía al final de la presente nota, aparte 166

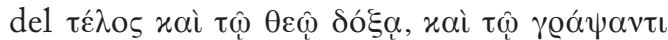
(«fin, gracias a Dios y al escribiente»), que cierra el manuscrito, se pueden leer dos anotaciones cronológicas de redacción. La primera, anotada originalmente por la mano del copista en numeración arábiga, indica la fecha de redacción del manuscrito, mientras que la segunda es una anotación anónima, posterior y escrita a lápiz en la que se especula la lectura de la anotación original: 1825 ஜे I828; Es decir, «نI825 ó I828?» que son también las dos alternativas que of rece la descubridora del manuscrito en su primera lectura (Omatos, 2006: I73), porque como bien indicaba ella en su trabajo, «no se distingue con claridad la última letra». En efecto, el trazo descuidado del último carácter de la fecha se presta a confusión y presenta cierta dificultad de lectura. Sin embargo, un cotejo entre el colofón y las páginas del manuscrito cuyas fotografías adjunta $\mathrm{O}$. Omatos en su estudio, nos permite comprobar que la grafía empleada habitualmente por el copista para la $\eta$ es inconfundible y no coincide, en absoluto, con el trazo de este último carácter de la fecha del colofón. Dado también que debemos descartar de antemano las otras lecturas inicialmente posibles $\alpha$ «I», $\beta$ «2», $\gamma$ «3», $\delta$ «4» y $\theta$ «9», debido a que el copista emplea también en estos casos una grafía completamente distinta, sólo quedan tres posibles lecturas probables $\varepsilon$ « ${ }^{\prime}, \varsigma$ «6» y $\zeta$ «7». La diversidad de trazos con que el copista escribe la $\varepsilon$ podría explicar el trazado inseguro con que fue escrito este último carácter, sin embargo ninguna de las variantes gráficas que se observan en el manuscrito se identifica con ésta. Asimismo la grafía de $\zeta$ que se observa en el manuscrito, tampoco parece corresponderse con este último. Así pues, en espera de una futura autopsia

del manuscrito, de estas tres probables lecturas nos decantamos, aunque con reservas, por la $\varsigma$ «6», de modo que la datación del manuscrito, según la lectura que proponemos para la fecha del colofón, sería el año I826. Otros datos relativos a la procedencia del manuscrito, como la identidad de su primer propietario o del copista, contribuiría a dilucidar con más exactitud el año en que fue redactado este manuscrito, pero desafortunadamente aún nos son desconocidos.

$\mathrm{Al}$ igual que los otros tres manuscritos y la tercera edición de la versión intermediaria de Lorenzo Franciosini (Roma 1677), el manuscrito n. ${ }^{\circ} 798$ de la Biblioteca Yenadios omite de forma sistemática la traducción de las composiciones versificadas del original español. Asimismo el registro lingüístico en que está escrito el manuscrito, parece identificarse, a simple vista, con la misma variedad mixta de rasgos cultos y vernáculos del dialecto fanariota (constantinopolitano), en que están escritas las otras tres copias. En el plano léxico, se observa gran parte de los mismos rasgos italianizantes, como

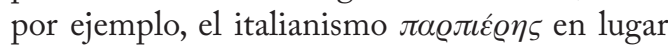
del griego rov@éas 'barbero', la traducción o la transcripción «a la italiana» de nombres propios,

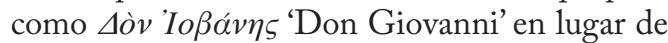

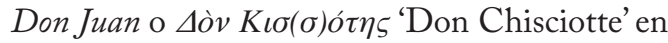
lugar de Don Quijote. Independientemente de las correcciones apreciadas (Kejayoglu, 2009: Io) en la transcripción de los títulos de cada uno de los capítulos (Omatos, 2006: I73-179), éstos coinciden, en su mayor parte, con los títulos de los capítulos del códice n. ${ }^{\circ}$ I47r de la Biblioteca de la Academia Rumana. Otro rasgo coincidente con este tercer manuscrito es el uso grafemático de un punto sobre las consonantes oclusivas sordas bilabial $\langle\pi\rangle$ y dental $\langle\tau\rangle$ para notar su correspondiente sonora [b] y [d], en lugar de notarlas con los respectivos dígrafos $\langle\mu \tau\rangle$ y $\langle v \tau\rangle$, como convencionalmente se hace 
en el griego actual. A la espera de que este cuarto manuscrito sea editado pronto, todo parece indicar que este cuarto manuscrito pertenece a la misma tradición que los otros tres manuscritos (Kejayoglu, 2009: ro).

A pesar de que Y. Kejayoglu y A. Tabaki han dilucidado datos, como la datación aproximada de los manuscritos o algunos de sus propietarios, la identidad del traductor, sin embargo, aún nos sigue siendo desconocida. La rúbrica de la «domna»4 Smaragda (Esmeralda) Stavropóleos, la tercera esposa de Nicolás Mavrocordatos, que se puede leer en la última página del manuscrito ateniense de la familia Sgurdeos, indujo a su descubridora a dar por sentado que la cónyuge del voivoda fanariota era la auténtica autora de la traducción (Drulia, I966: 26). Sin embargo a tenor de los últimos avances de la investigación en torno a la lengua y el estilo de la traducción (Kejayoglu - Tabaki, 2007: * $\left.{ }^{3} 35^{-*} 49\right)$, la hipótesis más probable es que la domna Smaragda fuera, en realidad, la propietaria originaria del manuscrito y no su autora. El registro lingüístico semiculto fanariota del traductor, el sustrato de lengua culta eclesiástica que se observa aquí y allá en la traducción, así como determinadas huellas fonéticas de los dialectos neogriegos septentrionales podrían ser indicativos del origen e identidad del traductor, el cual da muestras de conocer bien el latín y el italiano, pero no tanto. Según se desprende de los errores de interpretación, las omisiones, las abreviaciones y las perífrasis que incluye en el texto, debemos excluir la posibilidad de que sea un italiano que domine el griego culto eclesiástico y hable la variedad dialectal fanariota.

El estilo del anónimo traductor no parece

$4 \Delta o ́ \mu v a(<$ rum. doamna < lat. domina) es el título nobiliario moldo-valaco con que se denomina a la esposa del voivoda. identificarse con el de otros traductores contemporáneos conocidos, como Ioanis Avramios o Dimitrios Procopios Páberis. Su traducción lejos de ser una versión libre o una recreación de la traducción de Franciosini, es una fiel interpretación de ésta. Este modus operandi es característico de las traducciones griegas dieciochescas, vinculadas a la tradición de dragomanes y voivodas fanariotas y del pequeño círculo de intelectuales políglotas que los rodeaba, aficionados a clásicos literarios de contenido filosófico, ético, moral y político. A tenor de todos estos indicios, la identidad del traductor hemos de buscarla en el entorno y en seno de la familia de los Mavrocordatos: el propio Nicolás Mavrocordatos, su yerno y consejero, Juan Escarlato, su hijo primogénito Escarlato y el médico de la familia Tomás Testabuzas. Desgraciadamente, hasta el momento no disponemos de ningún testimonio ni indicio concluyente que nos permita identificar, con total seguridad, el anónimo traductor. Sin embargo, el incipiente interés por el Quijote que reconocen abiertamente Escarlato Mavrocordatos y Tomás Testabuzas en la correspondencia epistolar, que mantuvieron entre finales de 1720 y comienzos de I72I (Papacostea-Danilopolu, I990: 29-37), nos incita a conjeturar que muy probablemente uno de estos dos sea el autor de la traducción. De hecho, a tenor de las palabras de Testabuzas a propósito de la universal obra cervantina, de la que dice que es «un libro divertidísimo, para el cual yo tengo también una comedia muy bonita, para narrar con ingenio las locuras de aquellos que, sin conocerse a sí mismos, fantasean con vanidades» (Papacostea-Danilopolu, 1990: 37), no parecería excesivamente aventurado atribuirle a este último la autoría de esta primera traducción griega del Quijote.

Respecto a la edición de Y. Kejayoglu, como ya hemos dicho, ésta contempla sólo los tres 
primeros manuscritos hallados: el pertenenciente a la familia Sgurdeos, el n. ${ }^{\circ}$ I de la Biblioteca Municipal de Vitina y el n. ${ }^{\circ}$ I47 I de la Biblioteca de la Academia Rumana (KejayogluTabaki, 2007: *93-"'193). Se trata de una edición filológica de uso, ${ }^{5}$ que corrige las distorsiones, alteraciones e imperfecciones introducidas en el texto que transmiten los tres manuscritos (recordemos que ninguno de ellos es autógrafo del traductor original), pero que está dirigida al gran público no especializado en cuestiones filológicas. Los principios y las pautas ortográficas y grafemáticas que el editor ha seguido a la hora de establecer el texto definitivo (Kejayo-

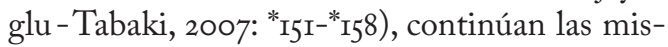
mas prácticas ecdóticas que Y. Kejayoglu viene aplicando en otras ediciones crítico-filológicas de textos literarios griegos tardo-medievales y modernos, como la Crónica de Chipre de Jorge Vustronio (Nicosia 1997), la novela en verso de Apolonio de Tiro (Salónica 2004) o las dos antologías de prosa neogriega (Atenas $1999 \mathrm{y}$ Salónica 200I). Al igual que estas ediciones, la presente tiene como característica principal la normalización y la regularización ortográfica y acentual de los usos grafemáticos, que transmiten los tres manuscritos, de acuerdo a la gramática vigente del griego moderno común (demótico) actual, a los usos grafemáticos y ortográficos actuales y al sistema diacrítico monotónico. Aun partiendo de esta premisa, el editor no «corrige» en ningún momento los fenómenos

5 No es una edición crítica en el sentido estricto de la palabra, porque los tres manuscritos que considera transmiten pasajes distintos de la traducción y en ningún momento se precisa la colación o comparación de manuscritos. No obstante, con el hallazgo del último manuscrito n. ${ }^{\circ} 798$ de la Biblioteca Yenadios de Atenas, que transmite por completo la segunda parte del Quijote, si se podrá establecer una edición propiamente crítica colacionando el texto de este manuscrito con el que transmite el códice $n$. $^{\circ}$ I47I de la Biblioteca de la Academia Rumana.

fonéticos de los dialectos septentrionales que continuamente se observan en el texto de los manuscritos. Asimismo conserva determinadas convenciones ortográficas y grafemáticas, que resultan indispensables para la distinción de formas gramaticales cultas: como por ejemplo, algunos nombres propios ('A $\mu \alpha \delta \iota \varsigma$ 'Amadís'), la iota suscrita en el dativo ( $\varepsilon v \tau \alpha v \tau(\dot{)})$, el apóstrofe en la crasis $\left(\alpha \pi^{\prime} \varepsilon \delta \omega^{\prime}\right)$, la tilde en eventuales disílabos $(\delta \iota \alpha ́)$ o el acento de énclisis en eventuales sintagmas de sustantivos trisílabos o de más

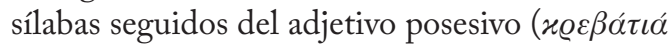

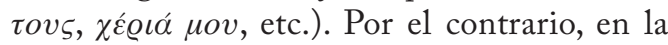
edición sí se corrigen tácitamente algunos usos grafemáticos y ortográficos que se observan en la tradición textual griega manuscrita e impresa de esta época, pero que son infundados desde el punto de vista etimológico o fonético: como por ejemplo, el empleo de la geminada $\langle\lambda \lambda\rangle$ por la simple $\left\langle\lambda>\right.$ (el aoristo de $\beta \alpha^{\prime} \lambda \lambda \omega, \ddot{\varepsilon} \beta \alpha \lambda \lambda \alpha$ por $\left.\dot{\varepsilon}^{\prime} \beta \alpha \lambda \alpha\right)$, la sustitución sistemática de $\langle\tau \sigma\rangle$ por $\langle\tau \xi\rangle$ en posición inicial, media o final de palabra (

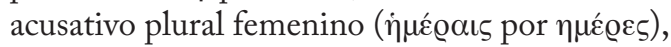
el uso redundante de $\langle\gamma\rangle$ o $\langle\gamma l\rangle$ para notar la

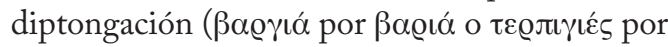
$\tau \varepsilon \varrho \pi(\gamma \hat{s})$ ). Asimismo en la edición se normaliza tácitamente la puntuación del texto, la separación por parágrafos y la introducción del estilo directo, teniendo como modelo la edición del original español preparada por Francisco Rico y Joaquín Forradillas para el Instituto Cervantes en la conmemoración del cuarto centenario de la primera edición de la primera parte del Quijote (Barcelona 2004).

Pese a que Y. Kejayoglu advierte en su nota (Kejayoglu - Tabaki, 2007: *157) que su edición no ambiciona la enmienda de todos y cada uno de los errores o distorsiones que haya introducido en el texto la transmisión manuscrita, la edición consta de un aparato crítico en el 
que se registran no sólo la correcta lectura o las propuestas de enmienda de muchos pasajes confusos de la traducción, sino también las intervenciones y correcciones que el propio editor introduce: como por ejemplo, las múltiples notaciones de sonorización de consonantes sordas que transmiten los manuscritos $(\langle\pi\rangle\rangle$ $\langle\mu \pi>$ para la sonora $[\mathrm{b}])$ o el empleo de gemi-

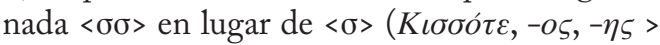

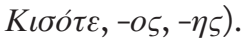

Como vemos, la edición del profesor Kejayoglu es realmente compleja -diríamos incluso que controvertida en algún punto-, debido, por una lado, a las distorsiones inherentes a la transmisión textual manuscrita, y por otro, al polimorfismo y a la fluctuación entre variantes cultas arcaizantes y vernáculas, tan característica de la lengua griega de este período. Esta "poliglosia» de la lengua griega de la época no puede dejar de reflejarse en la tradición de textos griegos tanto impresos como manuscritos producidos entre I 453 y I82I, que presentan una serie de usos grafemáticos y ortográficos irreconocibles para los lectores griegos actuales. Es por ello que el profesor Kejayoglu ha optado por las convenciones lingüísticas del griego demótico y monotónico actual, para establecer un texto formalmente más cercano y más accesible para el gran público griego. En este sentido, si el objetivo final que se marca Kejayoglu en su edición es facilitar al lector medio griego actual la lectura de texto griego, como éste, de la tercera década del siglo XVIII, podemos afirmar sin tapujos que la edición logra con éxito la meta marcada. Ahora bien, cuando Kejayoglu establece el texto guiado por criterios más reguladores que historicistas, irremediablemente se aleja de la eventual forma inicial del texto arquetípico, de tal modo que si lo leyera su anónimo autor original, seguramente tendría dificultades para comprenderlo, de la misma manera que el lec- tor medio griego actual difícilmente alcanzaría a entender el texto arquetípico original. En realidad, éste es uno de los dilemas que ha de afrontar la ecdótica moderna de textos griegos (fundamentalmente vernáculos) manuscritos e impresos entre los siglos XV y XIX: reconstruir con fidelidad histórica el texto supuestamente original o normalizarlo y adecuarlo a los usos grafemáticos y ortográficos actuales, para facilitar su lectura. En el difícil equilibrio entre estos dos extremos, Y. Kejayoglu se decanta en la presente edición por el segundo.

Llegados a este punto, no queríamos desaprovechar la ocasión, para señalar dos observaciones a propósito del texto editado por Kejayoglu. Por un lado, creemos que en la edición debería evitarse la multiplicidad de variantes de un mismo nombre propio que transmiten

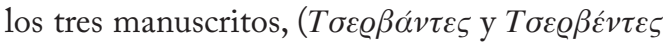

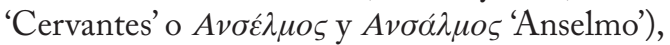
adoptando siempre para cada caso la forma lingüística o etimológicamente más correcta

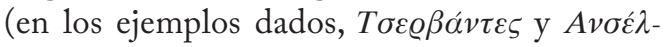
$\mu o \varsigma)$. Por otro lado, por razones estrictamente historicistas nos hubiera parecido más adecuado emplear el sistema diacrítico politónico, toda vez que éste no dificulta en absoluto la lectura actual del texto y ofrece en contrapartida una imagen gráfica y visual del texto más cercana al original manuscrito. En cualquier caso, la edición establecida por el profesor Kejayoglu no deja de ser, en absoluto, una edición filológica de uso muy bien cuidada y con un exhaustivo aparato crítico que satisface las exigencias de los lectores más duchos en cuestiones filológicas y ecdóticas.

La aparición del nuevo manuscrito n. ${ }^{\circ} 798$ de la Biblioteca Yenadios lejos de invalidar el excelente trabajo de Y. Kejayoglu y A. Tabaki, lo actualiza y abre nuevos horizontes en la investigación sobre el proceso inicial de recepción y 


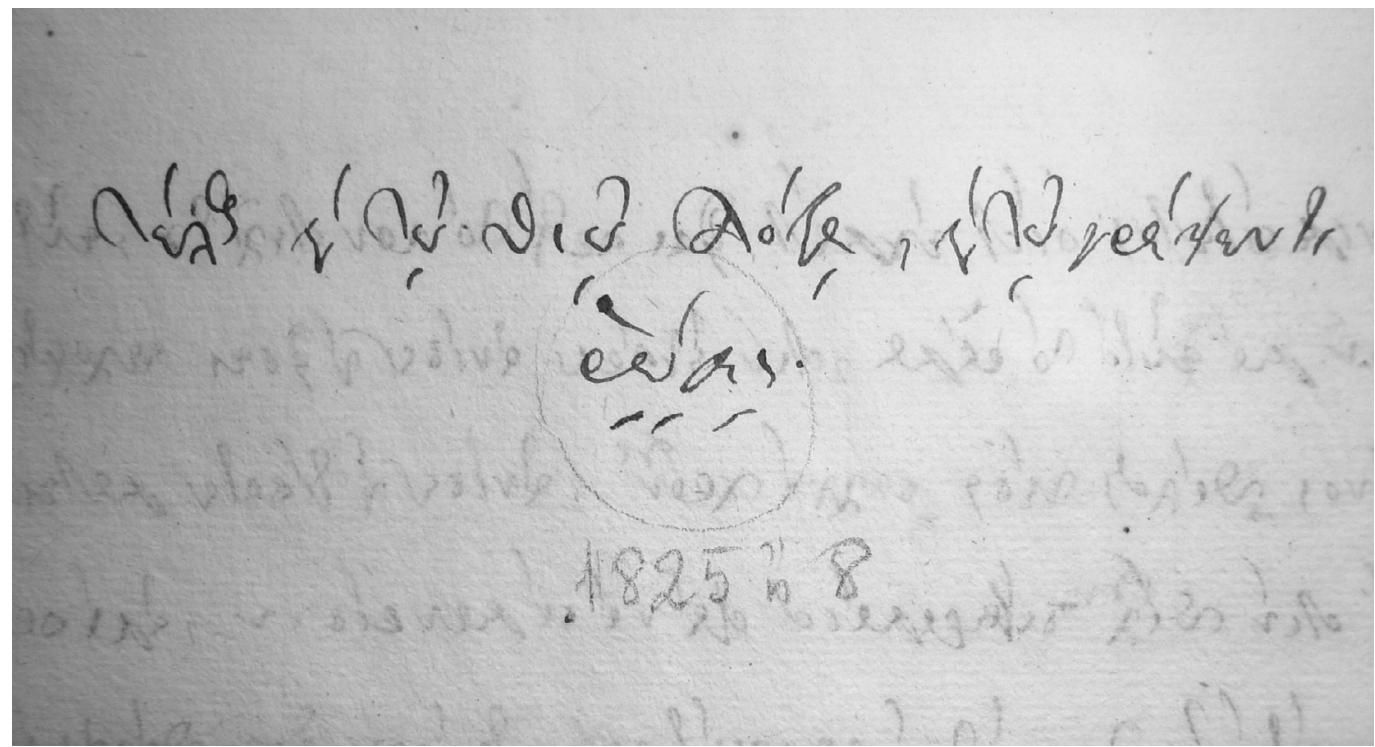

Colofón del ms. 798 de la Biblioteca Yenadios de Atenas

difusión del Quijote en el ámbito griego, balcánico y otomano del siglo XVIII. Es por ello que se hace absolutamente precisa la edición y el análisis lingüístico y paleográfico de este nuevo testimonio manuscrito, que tal vez nos ayudará a resolver algunos de los interrogantes que aún nos plantea este primer Quijote traducido al griego en la Bucarest del siglo XVIII.

RECIBIDO EN NOVIEMBRE DE 2009 ACEPTADO EN DICIEMBRE DE 2009 VERSIÓN FINAL DE ENERO DE 2010

\section{REFERENCIAS BIBLIOGRÁFICAS}

Cervantes Saavedra, Miguel de (1677)². L'ingegnoso Cittadino Don Chisciotte della Mancia. Trad. Lorenzo Franciosini. 2 vols. I6 grabados. Roma: nella Stamperia di Giuseppe Corvo.

- (I799). Don Quichotte de la Manche. Trad. JeanPierre Claris de Florian. 3 vols. París: Deterville.

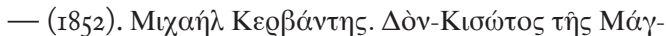

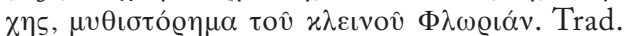
Ceódoros Catramís. 2 vols. Esmirna: 'Iovıxòv

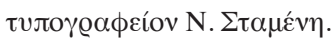

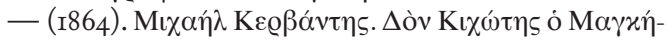

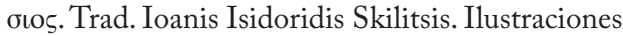

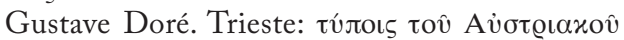
$\Lambda$ óvio,

- (2004). Don Quijote de la Mancha, edición del Instituto Cervantes I605-2005, estudio preliminar F. Lázaro Carreter, ed. F. Rico y J. Forradillas Barcelona: Galaxia Gutenberg - Círculo de Lectores - Centro para la edición de los clásicos españoles, 2004.

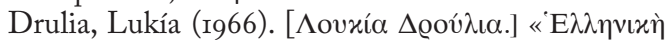

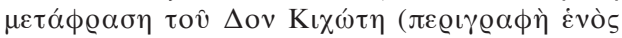

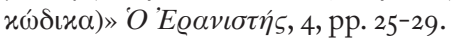

Ivanovici, Victor (2009): «Grecia». Gran Enciclopedia Cervantina. Director Carlos Alvar. Vol. VI. Madrid: Castalia-Centro de Estudios Cervantinos, pp. 5478-5487.

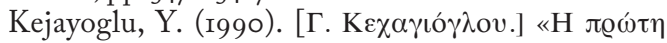

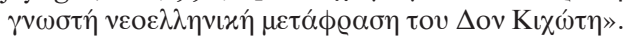

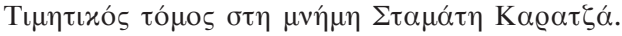
Salónica, pp. I75-84. 


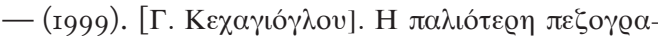

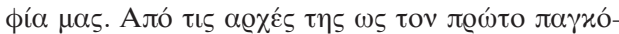

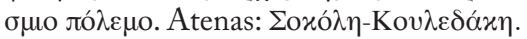

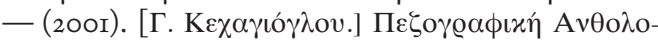

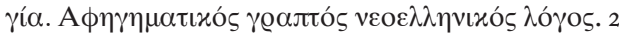

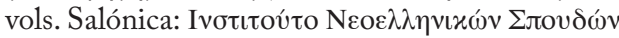

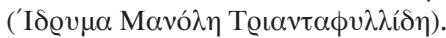

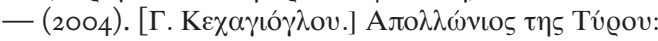

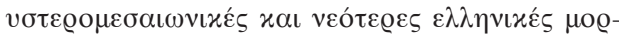

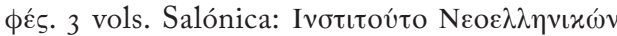

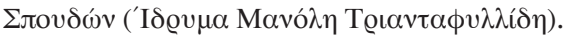

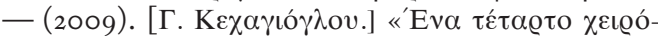

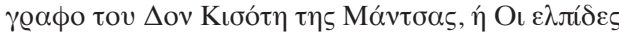

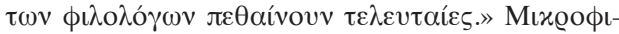

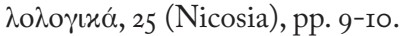

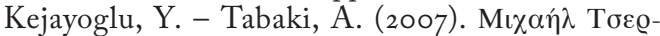

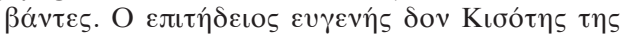

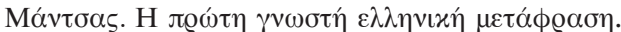
Introd. Y. Kejayoglu y A. Tabaki. Ed., vocabulario

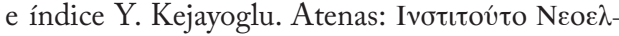

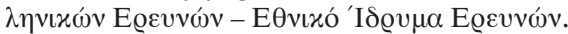

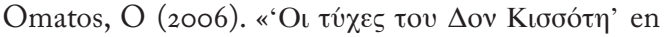
un nuevo manuscrito del siglo XIX». Erytheia, 27, pp. $167-185$.

Papacostea-Danilopolu, Cornelia (I990). «Préoccupations livresques de Scarlat Mavrocordat dans un manuscrit de l'Académie Roumaine». Revue des Études Sud-Est Européennes, 28, pp. 29-37.

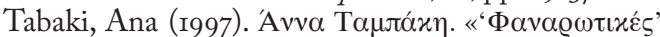

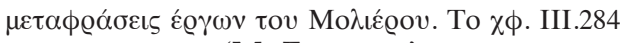

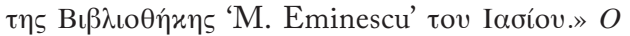

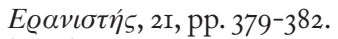

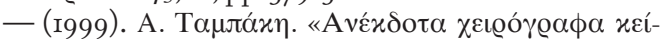

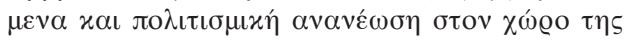

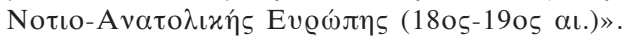

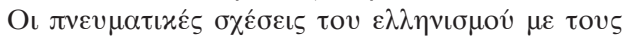

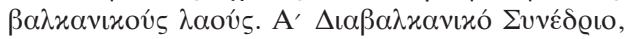
Comotiní, pp. I99-210.

Villar Lecumberri, Alicia (200I). «La presencia de Cervantes en los manuales griegos». Volver a Cervantes. Actas del IV Congreso Internacional de la Asociación de Cervantistas (Lepanto, I-8 de octubre de 2000). Ed. A. Bernat Vistarini. Vol. I. Palma de Mallorca: Universitat de les Illes Ballears, pp. 75-90.

- (2008). «Huellas cervantinas en la poesía neohelénica». Tus obras los rincones de la tierra descubren. Actas del VI Congreso Internacional de la Asociación de Cervantistas (I3-I6 de diciembre de 2006). Ed. Alexia Dotras Bravo, José Manuel Lucía Megías, Elisabet Magro García y José Montero Reguera. Alcalá de Henares: Asociación de Cervantistas Centro de Estudios Cervantinos, pp. 789-797.

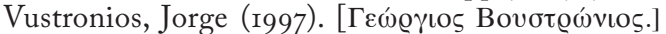

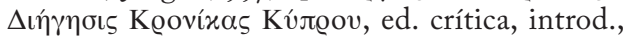

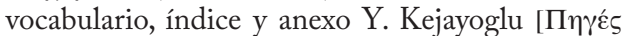

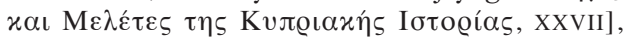

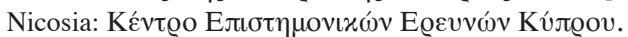

